\title{
Pressure Measurement of the Flexible Pipe Algorithm in Aerial Refueling based on SVR with Parameter Optimization
}

\author{
Wanxu Zhang ${ }^{1, ~ a ~}$, Xiangnan $\mathrm{Li}^{1, \mathrm{~b}}$ and Lin Wang ${ }^{1, \mathrm{c}}$ \\ ${ }^{1}$ School of Information Science and Technology, Northwest University, Xi'an, China.

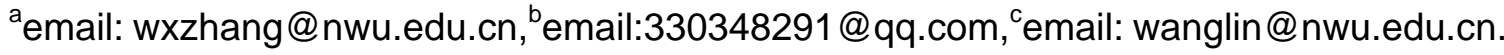

Keywords: aerial refueling; flexible pipe pressure; SVR; parameter optimization

\begin{abstract}
With the problem that the pressure of the flexible pipe in aerial refueling was hard to be measured directly by sensors, a pressure measurement algorithm based on support vector regression (SVR) with parameter optimization was proposed in this paper. First, a data model with a large amount of historical data based on SVR was set up. Then the parameters of the model were optimized by cross-validation and grid-search method. Experimental results show that the method has a high prediction precision and good generalization ability comparing with BP neural network.
\end{abstract}

\section{Introduction}

Aerial refueling is a process of transferring fuel from one aircraft to another during flight, which can increase the cruise range and active radius of a fighter [1,2]. For many years, great progress has been made in aerial refueling by continuous technical innovation and application. Mao W and Eke FO summarized the key technology of the probe drogue aerial refueling systematically [3]. Dong X analyzed the progress of essential procedures researches in aerial refueling [4]. Lu Y summarized the advances in the dynamical modeling and control of aerial refueling based on the consulting to a large amount of references [5].

To ensure the safe of refueling, parameters, such as pressure and flow, must be measured in real time. In general, the system gathers information by sensors and devices which are mounted directly on fuel line. When an aircraft flies in atmosphere, it cannot decorate signal cables or sensors to measure pressure directly because of the effect of atmosphere disturbance [6]. At present, the Venturi tube, which is installed in the refueling system, is used to measure pressure. However, it is expensive and difficult to install. Through analyzing the components and principle of the refueling system, a pressure measurement method based on support vector regression (SVR) with parameter optimization is proposed in this paper. And the nonlinear relationship between flexible pipe pressure, metal pipe pressure and flow with a large amount of historical data is established. Experimental results show that the method has a high calculation accuracy and good generalization ability comparing with back propagation (BP) neural network.

\section{Principle of Aerial Refueling System}

The fuel line of an aerial refueling system is composed of metal pipe and flexible pipe. The system structure is shown in Fig. 1.With the pump pressure, the fuel flows into the flexible pipe through metal pipe. The control device uses fuel pressure and flow to monitor and control the whole system during fueling.

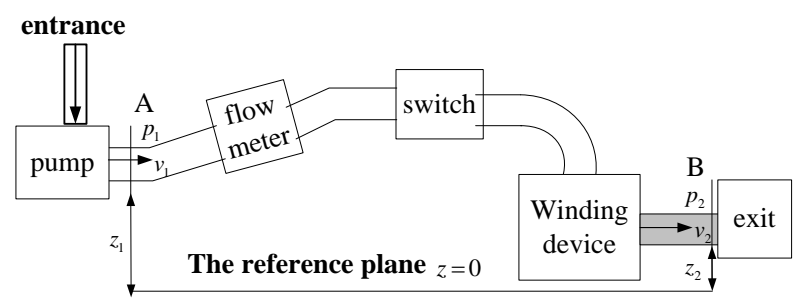

Fig.1. The structure of aerial refueling system 
In Fig.1, slow variable cross-section A and B are on the metal pipe and at the end of flexible pipe, respectively. Where $p_{1}$ and $p_{2}$ are the pressure of section $\mathrm{A}$ and $\mathrm{B}, \mathbf{V}_{1}$ and $\mathbf{V}_{2}$ are the average flow of the two sections. The refueling system conforms to the theory of fluid mechanics. The continuity equation and Bernoulli equation are as follows:

$$
\begin{aligned}
& Q=S_{1} \mathbf{V}_{1}=S_{2} \mathbf{V}_{2} . \\
& \frac{\alpha_{1} \mathbf{V}_{1}^{2}}{2 g}+z_{2}+\frac{p_{1}}{\rho g}=\frac{\alpha_{2} \mathbf{V}_{2}^{2}}{2 g}+z_{2}+\frac{p_{2}}{\rho g}+h_{L T} .
\end{aligned}
$$

where $S_{1}$ and $S_{2}$ are the area of low variable cross-section A and B, which are known. $Q$ is fuel flow measured by flow meter installed in the pipeline. $\alpha_{1}$ and $\alpha_{2}$ are the representation of the kinetic energy correction factor of the two sections. $z_{1}$ and $z_{2}$ indicate the height relative to the reference plane of the two sections. $\rho$ is the fuel density and $h_{L T}$ is the hydraulic loss.

From formulas (1) and (2), we have

$$
p_{2}=p_{2}+\frac{\rho}{2}\left[\alpha_{1}\left(\frac{Q}{s_{1}}\right)^{2}-\alpha_{2}\left(\frac{Q}{S_{2}}\right)^{2}\right]+H \rho g-h_{L T} \rho g \text {. }
$$

According to formula (3), there is a nonlinear relationship between $p_{1}, p_{2}$ and $Q$.After calculating $h_{L T}, p_{2}$ can be deduced by $p_{1}$ and $Q$ which can be measured by sensors.

\section{Pressure Measurement of the Flexible Pipe Model in Aerial Refueling based on SVR}

The support vector machine (SVM) is an algorithm based on structure risk minimizing principle and high generalization ability, which was presented by Vapnik in 1995. Support vector regression (SVR) is an important branch of SVM. SVR has been applied to system identification, nonlinear system prediction and good results have been demonstrated. After analyzing components and principal of aerial refueling system in previous section, flexible pipe pressure measurement is a nonlinear regression problem. And in this paper a pressure measurement method of support vector regression (SVR) with parameter optimization is proposed.

In the pressure measurement of the flexible pipe model, the sample set is $\left\{\left(x_{i,} y_{i}\right)\right\}, i=1,2 \ldots n$. Where $x_{i}$ is input variable, which consists of metal pipe pressure $p_{1}$ and flow $Q$, and $y_{i}$ is output variable, which is flexible pipe pressure $p_{2} . n$ is number of samples. The overall design scheme is shown in Fig.2. First, in order to improve the quality of the data, the sample data should be preprocessed. Second, the flexible pipe pressure measurement model based on SVR is established. Third, the model parameters are optimized in order to improve the generalization of the model. Then the model is corrected with the experimental data to increase its accuracy. Finally, substitute $p_{1}$ and $Q$ into the model to calculate $p_{2}$.

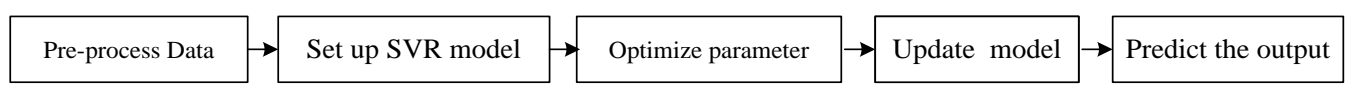

Fig.2.The overall design scheme

Pressure Measurement of the Flexible Pipe Model based on SVR. In the paper, building the measurement model means to define a function $f$. Though training the coefficient of function with historical data, the model will be set up.

(1) Define the function

The problem of pressure measurement is a nonlinear optimization problem. Based on the principle of SVR, the method can translate the nonlinear features in the original space to linear features in the high-dimension state space. The linear function is the function of SVR, which is as follows.

$$
f(x)=w \llbracket \varphi(x)+b, \varphi: R^{n} \rightarrow F, w \in F .
$$

where $w$ is the regression coefficient, $b$ is the threshold. 
(2) Estimate $w$ and $b$

$w$ and $b$ are calculated by using penalty function method.

$R(C)=\frac{1}{2}\|w\|^{2}+R_{\text {emp }}=\frac{1}{2}\|w\|^{2}+\frac{C}{n} \sum_{i=1}^{n}\left|y_{i}-f(x)\right|_{\varepsilon}$, where $|y-f(x)|_{\varepsilon}=\left\{\begin{array}{l}0,|y-f(x)| \leq \varepsilon \\ |y-f(x)|-\varepsilon, \text { else }\end{array}\right.$

where $C$ is the penalty factor, and $\varepsilon$ is the maximum error between prediction and testing, which is called non-sensitivity coefficient. And the algorithm is called $\varepsilon-S V R$.

To solve the equation, the problem (5) is described as a constraint optimization problem [7].

$$
\begin{gathered}
\min _{w, b} \frac{1}{2}\|w\|^{2}+C \sum_{i=1}^{n}\left(\xi_{i}+\xi_{i}^{*}\right) \quad \text { s.t. } y_{i}-w^{T} \varphi(x)-b \leq \varepsilon+\xi_{i}^{*} \\
w^{T} \varphi(x)+b-y_{i} \leq \varepsilon+\xi_{i} \\
\xi_{i}, \xi_{i}^{*} \geq 0
\end{gathered}
$$

where $\xi_{i}$ and $\xi_{i}^{*}$ are the attenuation factors, which are corresponded to each sample. With the equivalent problem, the Lagrangian multiplier method is finally employed to obtain the optimum solution in an analytical form to the original problem.

Parameter Optimization. To obtain a good generalization performance, parameters, such as the penalty factor $C$ and parameter of the kernel function $g$, should be optimized in the algorithm when training the model[8]. The k-fold cross-validation method (K-CV) and grid search GS is used to avoid blindness and randomness in model parameter selection, and the prediction precision is improved.

(1) Search $(g, c)$ with GS. The GS algorithm to determine the minimum of a cost function with possibly multiple optima is to evaluate a grid over the parameter space and to pick the minimum. This procedure iteratively zooms to the candidate optimum. Based on the method, we can determine the range and steps of $(g, c)$ and generate mesh. Go through all points in the grid, and get all $(g, c)$.

(2) k-fold cross-validation. In k-fold cross-validation, the original sample is randomly partitioned into $\mathrm{k}$ equal size subsamples. Of the $\mathrm{k}$ subsamples, a single subsample is retained as the validation data for testing the model, and the remaining $\mathrm{k}-1$ subsamples are used as training data. The cross-validation process is then repeated $\mathrm{k}$ times (the folds), with each of the $\mathrm{k}$ subsamples used exactly once as the validation data. The k results from the folds can then be averaged (or otherwise combined) to produce a single estimation. Based on the method, we estimate the results of all $(g, c)$. The result, which makes the estimation maximum, is the best optimal parameter.

\section{Results}

In the ground experiment site, we obtain 15000 groups of data by sensors and choose 9400 groups of them as training set,and the rest are testing set. After many experiments, the radial basis kernel function is the best one. The value range of $g$ and $C$ are both $[-8,8]$, and steps are both 0.5 . Based on grid search and 5-fold cross-validation, the optimal parameters are $C=181.0193, g=0.022097$. At the same time, the mean square error is 0.0013425 .

To verify the accuracy and usefulness of the method which this thesis puts forward, we build a BP network model as a comparison. If there is enough neuron, a BP network can approximate any nonlinear functions with any precision. The input variables are $p_{1}$ and $Q$, and the output variable is $p_{2}$. Set up a three layer BP neural network with 4 neurons of hide layers. Max training time is 1000, learning rate is 0.5 , momentum factor is 0.9 , and training goal is 0.00004 .

Keep the defaults for $g$ and $C$ in the simple SVR model, Where $g=1 / 2, C=1$. After experiments, prediction results are as follows. 

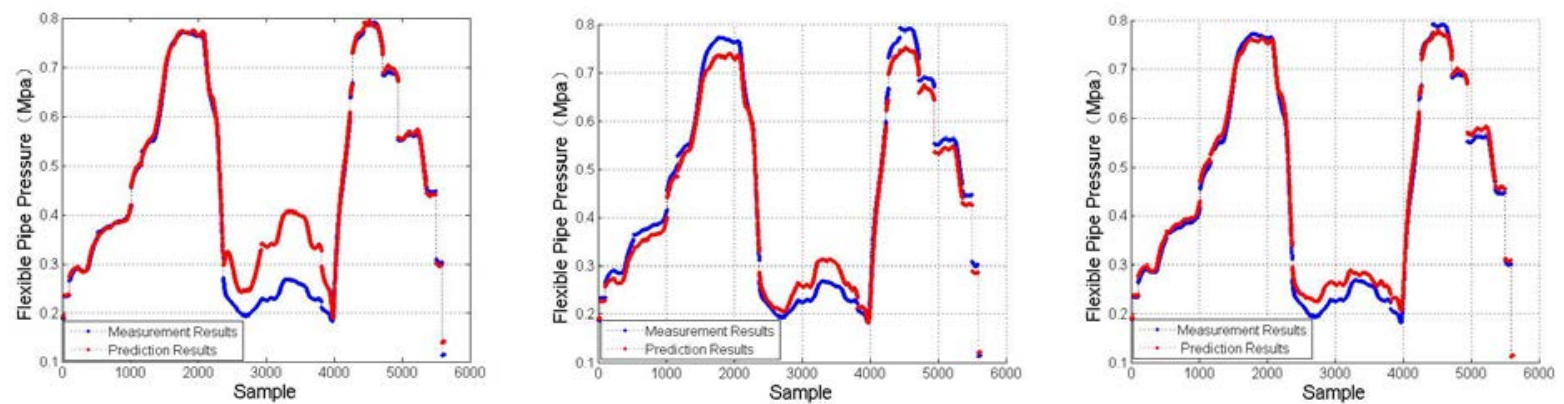

(a)BP neural network model (b)Simple SVR model (c)SVR model with parameter optimization

Fig.3 Results of different model

Observe the prediction results in Fig.3,the results of the BP neural network model is the worst, and the SVR model with parameter optimization is the best. The average relative errors of the models are shown in table1.

Table.1 Prediction results

\begin{tabular}{cccc}
\hline Model & BP neural network model & Simple SVR model & $\begin{array}{c}\text { SVR model with parameter } \\
\text { optimization }\end{array}$ \\
\hline $\begin{array}{c}\text { Average relative } \\
\text { error }\end{array}$ & $9.02 \%$ & $5.89 \%$ & $2.96 \%$ \\
Generalization ability & bad (over learning) & good & excellent \\
\hline
\end{tabular}

From Table.1,The average relative error of the SVR model with parameter optimization is $2.96 \%$, which is the best prediction result of the three models. Comparing the generalization ability of the models, there exists over learning in the BP neural network model and the SVR model with parameter optimization has excellent generalization ability.

\section{Conclusion}

This paper analyzes the working principle and the components of aerial refueling system. There is a nonlinear relationship between flexible pipe pressure, metal pipe pressure and flow. A support vector regression pressure measurement model with parameter optimization is set up. The method of cross-validation and grid-search is used to optimize the parameters of the model. After the comparison of BP neural network model, the simple SVR model and the SVR model with parameter optimization, the pressure measurement of the flexible pipe model in aerial refueling based on SVR with parameter optimization has high precision and good generalization ability, which might provided it with good application in aerial refueling.

\section{References}

[1]Pedro J O, Panday A. International Journal of Applied Mathematics and Computer Science, 2013, 23(1):75-90.

[2]Kriel S C, Engelbercht J A A, Jones T. Aerospace Science and Technology, 2013, 29(1):296-304.

[3]Mao W, Eke F O. Nonlinear Dynamic System Theory, 2008, 9(4):375-388.

[4]Xinmin Dong, Yuejian Xu, Bo Chen. Journal of Air Force Engineering University, 2008, 35(6):1-5. (in Chinese)

[5]Yuping Lu, Chaoxing Yang. Acta Aeronautica ET Astronautica Sinica,2014, 35, 1-15.(in Chinese)

[6]Ro K, Kamman J W. Journal Guidance Control and Dynamics, 2010, 33(1):53-63.

[7]Mingwei Li, Hong W C, Haigui Kang. Neurocomputing, 2013, 99(1):230-240.

[8]LinKai Sun, Jiashan Jin. Systems Engineering and Electronics, 2011, 33(8): 1820-1823. 